\title{
Leak Detection in Pipelines Using Wavelet Transform and Cepstrum Analysis Methods
}

\author{
Mohd Fairusham Ghazali \\ Faculty of Mechanical and Automotive Engineering Technology \\ Universiti Malaysia Pahang \\ Pekan, Malaysia \\ fairusham@ump.edu.my
}

\author{
Gigih Priyandoko* \\ Department of Electrical Engineering, \\ University of Widyagama \\ Malang, Indonesia \\ gigih@widyagama.ac.id
}

*corresponding author: gigih@widyagama.ac.id

\begin{abstract}
Nowadays, a piping system is one of the important features in either home or industrial user. Leak in piping systems is a major operational problem around the world. Leaks result to loss in the fluid through the flow and automatically affect to the economy of the user. Objective of this research is utilizing the signal processing using Wavelet Transform and Cepstrum Analysis methods to leak detect in pipeline. After experiment has been completed, the data analysis process by using Matlab Software takes place. The result shows that the accuracy of the leak location detection is accurate with small error results below $10 \%$.
\end{abstract}

Keywords-leak detection, MDPE, wavelet transform, cepstrum analysis

\section{INTRODUCTION}

Nowadays, piping system is one of the important features in either home of industrial user. It is essential for transporting fluid from a point to another point. For example, for home user, piping systems functioning as carrier for the water from the damp to every home. Whereas, for industrial user, the fluid that is carried are from different type such as water, gas, oil and many more. But due to many reasons, leaks may occur in the pipelines. Leak in piping systems is a major operational problem around the world. Leaks result to loss in the fluid through the flow and this will automatically affect the economy of the user. In addition, if the fluid consist of fluid that is dangerous and have potential risk to the health, this will be a problematic issue that needed quick action. Leaks may occur due to poor quality and defective pipe materials, pipe breaks resulting from poor workmanship, operational errors such as excessive pressure, closing or opening valves rapidly, corrosion, leaking fittings and accidental or deliberate damage to fixtures [1], [2]. Detecting, locating and repairing these leaks become a hard task. In many cases, leakage receives little or no attention from consumers and there is often no leakage management program until an emergency occurs or when there is shortage. Different methods for leak detection have been developed. Generally, the traditional methods give low recognition efficiency, high false rates and poor localization accuracy. Although the leaks can be detected but the location of the leaks cannot be identified. To overcome that problems, many method has been develop such as mass balance, volume balance, pig based monitoring systems and many more.

Leakage detection method can be categorized into two different categories which is external and internal method. Based on the external method, the leak detection can be categorized based on their type of operation [3], [4]. There are three categories which is sound propagation which consists of acoustic emissions, ground penetrating radar. The second is visual observation which includes direct observation and pig based monitoring systems. The third is by using smell such as tracer injection. The objective of this research is utilizing the signal processing using the Wavelet Transform and the Cepstrum Analysis.

\section{Methodology}

The research is then progress by experiment analysis so that data can be collected. After it has been completed, the analysis process by using Matlab Software takes place. The results of the analysis are then being verified and validate. If there are error in the results, the experimental and analysis must be done repeatedly until a good result can be obtained. The experiment starting by designing the experiment which includes the method use to run the experiment. Then, the test rig and pressure transducer are prepared. After that, the software part such as NI-DAQ Max, Matlab Software and Daisylab software. The experiment part such as test rig, pressure transducer, and pump are being setup. Next, data collection takes place by running the water throughout the pipe. The data is then analyzed by using Matlab software.

\section{SIGNAL ANALYSIS METHOD}

Leakage in the pipelines is becoming a huge issue of the world. A quick and smart action should be taken in order to minimize the loss and effect from the leakage. A traditional 
method such as visual inspection can be operates but that method required a long time. It is also lack in accuracy and may lead to false leak detection. Through signal analysis, the leak can be detected and the location of the leaks can be located [5]. So, without needed of another method the leaks can be repaired in no time. Signal analysis is a method of analyzing the data from the piping system that can be obtained through sensor installed at the pipe.

The history of signal analysis is starting by using Fourier transform techniques. But this method has its own limitations. It can only detect the leaks but cannot identify the location and the time when the leaks occurred. So, in order to obtain an accurate and complete data of the leak, many techniques of signal analysis have been developed such as Wavelet Transform [6]-[8], Cepstrum Analysis [9]-[11], Empirical Mode Decomposition (EMD) [11]-[13], Ensemble Empirical Mode Decomposition (EEMD) [14], [15], and many more.

\section{A. Wavelet Transform}

The wavelet transform is the most recent or newest solution to overcome the shortcomings of the Fourier transform. This method uses a fully scalable modulated window to solves the signal-cutting problem. The window is shifted along the signal and for every position the spectrum is calculated. Then this process is repeated many times with a slightly shorter or longer window for every new cycle. The Continuous Wavelet Transform is defined as the sum over all time of the signal multiplied by a scaled, shifted version of the wavelets function where both the time and frequency windows can be changed [6], [7], [13]. The idea of this method is generally the same as the Short Time Fourier Transform (STFT), but the only different is that the width of the windows is continuously changes throughout the transform [1], [6]. Theoretically, it can be calculated through equation below:

$$
Q(a, b)=\frac{1}{\sqrt{\alpha}} \int_{-\infty}^{\infty} y(t) \varphi^{*}\left(\frac{t-b}{\alpha}\right) d t
$$

where the index $Q(a, b)=$ wavelet coefficients, $a$ and $b$ are the scale (dilation) and translation (position) parameters, $y(t)$ $=$ vibration response signal. The $\varphi^{*}$ is the complex conjugate of the basis function. Disadvantages of continuous wavelet transform are this method consumes longer time and more resources to operate.

\section{B. Cepstrum analysis}

Cepstrum analysis can be categorized as new method among the others. This method is developed because current method of locating the leakage is too complicated, imprecise and time consuming. By using the Cepstrum Analysis, the existence and position of very small leakage can be detected. The advantage of this method is that it is fast, accurate and cheap to employ [10], [11]. In addition, cepstrum analysis is easier to use as it only requires filtering using wavelet and cepstrum analysis using MATLAB. Compared to other method, the peaks are sharper and have more defined peak when using cepstrum analysis. The basic theory is that cepstrum is defined as Fourier transform algorithm. There are two types of cepstrum which is complex cepstrum and power cepstrum. Cepstrum analysis has been used in many areas of application such as automotive, oil and gas, instrumentation and many more. Example of cepstrum analysis is machine diagnostic in monitoring of gearbox and rolling element bearing vibrations, gearbox testing to detect early gear faults, bearing fault detection, echo detection and removal and speech analysis [9].

\section{EXPERIMENTAL DESIGN}

Transient method will be used as the main method in this study. Pressure transient happened when there is sudden change in flow by closing or opening the valve in the pipelines. This will create wave propagation along the pipelines and this propagation will be used as a medium to detect the leaks. The present technique of transient method is by using two sensors that have been oppositely placed between the leaks. Disadvantages of that method is that the operation needed two operates the two sensors. Furthermore, the noise also must be heard by both of the sensors in order for the leaks to be detected, so the distance between the sensors is important to successfully detect the leak.

To overcome the problem from the present technique, this research has proposed a method based on the present transient method. The equipment has been modified by using just only one sensor which is pressure transducer place at in front of the pipelines. The concept is that when the pressure transient travels down the pipe and there is contact with the leaks, a reflection of the signal is created. This signal will be collected by the pressure transducer. Then the data will be analyzed by using another method of signal processing. The advantages of this proposed method are that the accuracy on detecting leaks on quiet leaks can be improved.

The materials used for the experimental analysis are high density polyethylene (MDPE) pipe. The MDPE can be categorized in the thermoplastic section. It is odorless, tasteless, and nontoxic polymers which make it become a commonly material use in the industry. The high-density polyethylene replaces the galvanized iron which is difficult to join, higher in weight and more expensive.

The experimental tests were conducted using pipeline network as shown in schematic diagram in Figures 1-2. The pipes used are MDPE pipe with diameter 1 inch and half inch. To supply constant water pressure to the system, the inlet of the pipeline is connected to the water tank. To introduce the effect of leaks into the system, pin hole that controlled by valve has been installed at various location along the pipeline. To create pressure transient, solenoid valve is used. The signal is captured using the pressure transducer located near the solenoid valve. The pressure transducer is connected to the data acquisition at frequency $1 \mathrm{kHz}$ and sample size $1 \mathrm{k}$. Each run lasted for 1 minutes and 10 second. In order to filter noise from the signals, the discrete wavelet transform is use. Then, these filtered data will be analyzed using cepstrum technique. The output from the cepstrum is a series of peak and we can identify the location of the leak or features depending on the time of the peak. Figure 2 shows the design of the test rig by using Solid-work software.

\section{RESUlT AND DisCUSSION}

When raw data is collected, wavelet analysis will firstly take place. The data will be load on the wavelet toolbox of one dimensional in the MATLAB. Figure 3 shows the sampled data after being decompose. The type of wavelet uses to decompose the signal is db-3 with 5 levels because of its compatibility to the raw signal which looks like the noise mishmash. The raw data is being filtered or decompose into 
five levels. The main objective of using the wavelet analysis is to filter and remove noise from the data. Only when the noise from the data which is not needed is removes, the signal of the features will be clearly seen.

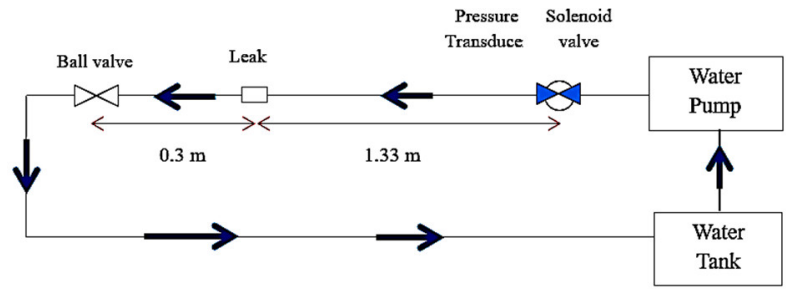

Fig. 1. Schematic diagram of test rig

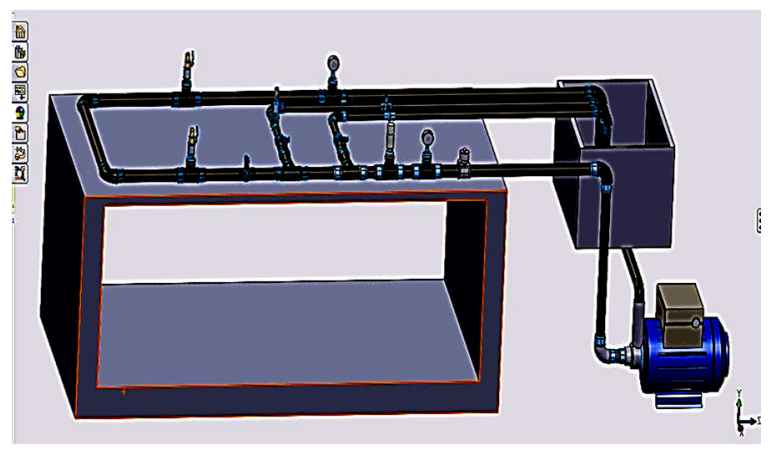

Fig. 2. Test rig

The process of removing the noise is called de-noising process. It is done by selecting the scaled white noise which exists in the data due to pump noise, surrounding noise and many other disturbances. Figure 4 shows the selection of scaled white noise for de-noising process. Figures 6-7 shows the original signal, de-noised signal and comparison between original and de-noised signal. From the figure, we can see that there is difference from before and after de-noised. The signal that is needed is clearly seen on the graph after being denoised. But the process of filtering and de-noising is depending on the amount of noise in the raw data. For example, if the noise is less the level of wavelet filtering should also be less and vice versa.

Cepstrum analysis will convert the wavelet data into the cepstrum data signal which show all the signal of the features located in the pipe. Speed of sound of this material is 950 $\mathrm{cm} / \mathrm{s}$. Figures 4-5 shows the pressure signal of raw data and cepstrum analysis of no leak and with leak. Figures 6-9 shows the pressure signal of the three conditions which is opening both leaks, opening only leak 1 and opening only leak 2 . The figure show that the signal from this material is noisy signal. This is due to the attenuation exists in MDPE pipe.

Attenuation is the combined effect of scattering and absorption. Signal amplitude will be reduced due to the spreading of the wave. Table 1 shows the summary data of trial first experiment by using MDPE pipe. MDPE pipe also absorbs the signal reflected by the features and thus weaken the signal. This will lead low signal or no signal of the features collected. Besides that, the error of the distance is quite high which are ranging from $2.88 \%$ to $16.1 \%$. Thus, it can be concluded that the accuracy of the leak location in MDPE pipe is small.

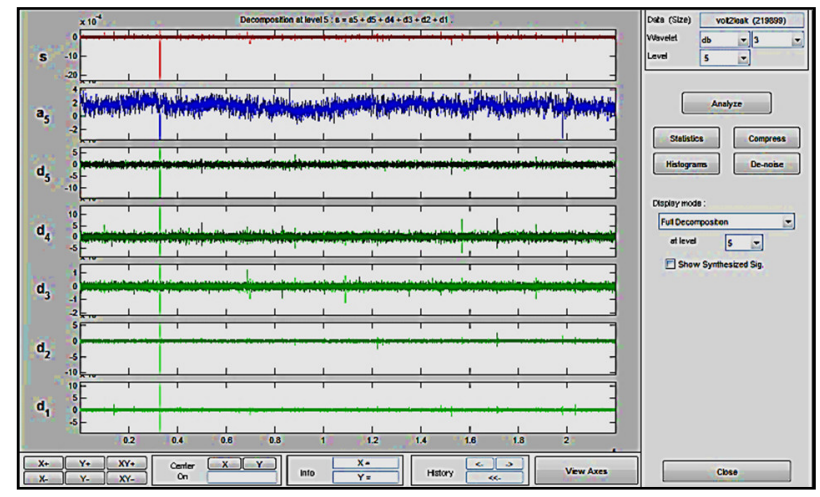

Fig. 3. Sampled data of wavelet filter and decompose

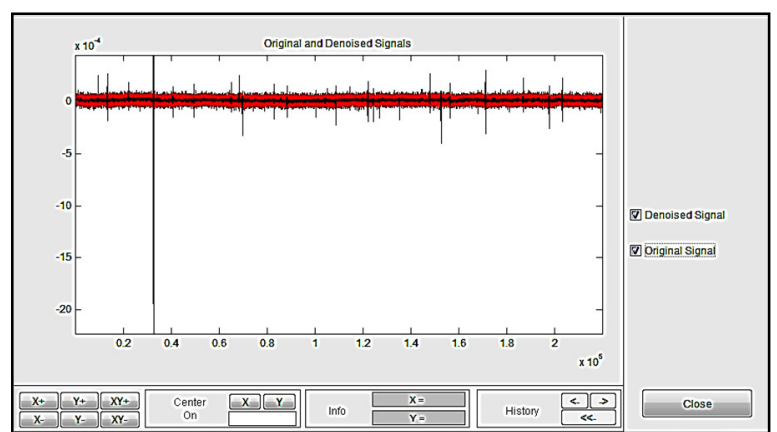

Fig. 4. Comparison between original (red line) and de-noised signal (black line)
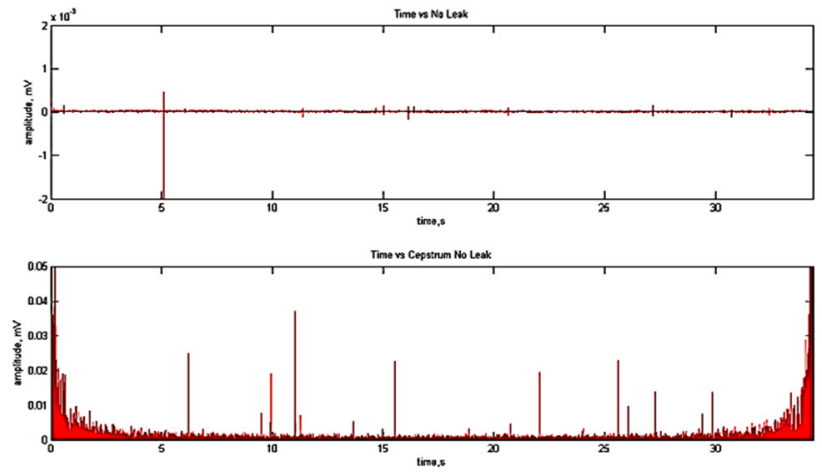

Fig. 5. Pressure signal of raw data and cepstrum analysis for no leak
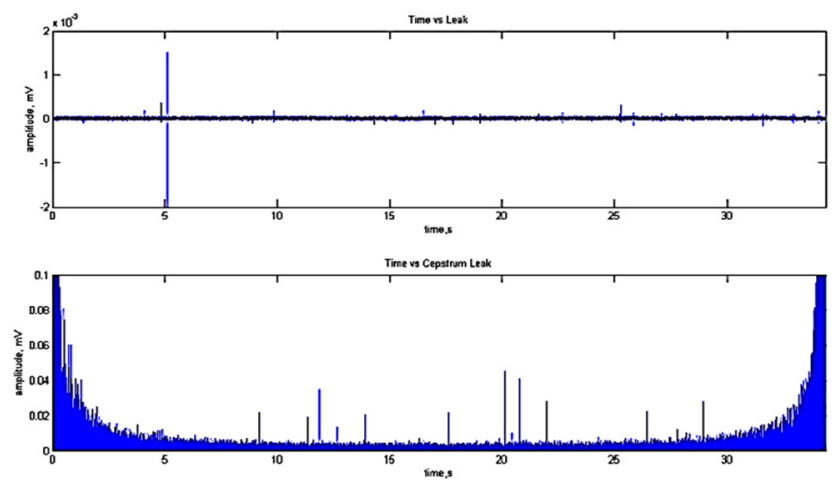

Fig. 6. Pressure signal of raw data and cepstrum analysis for leak 


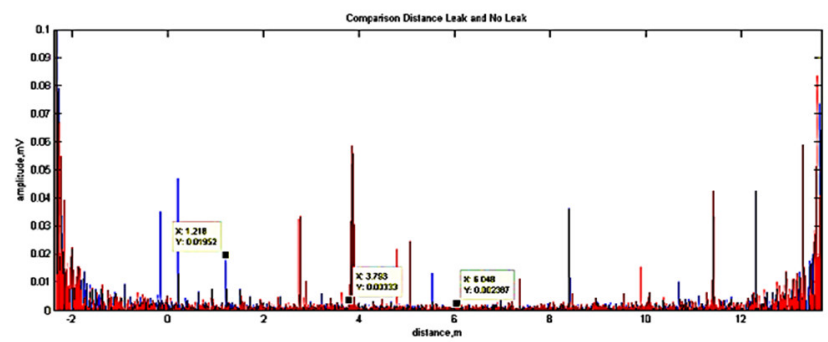

Fig. 7. Pressure signal of raw data and cepstrum analysis for leak

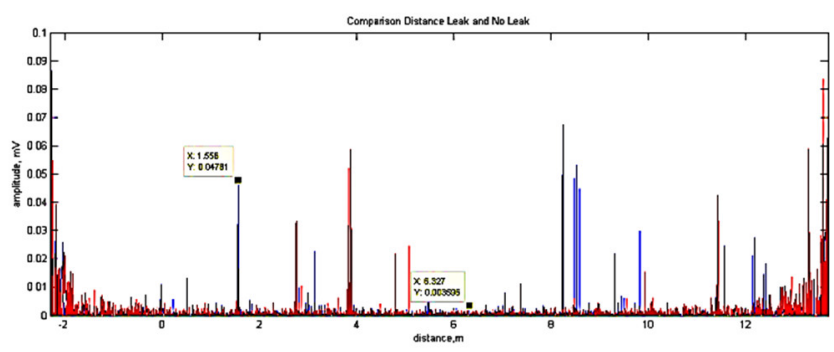

Fig. 8. Pressure signal when opening one leak

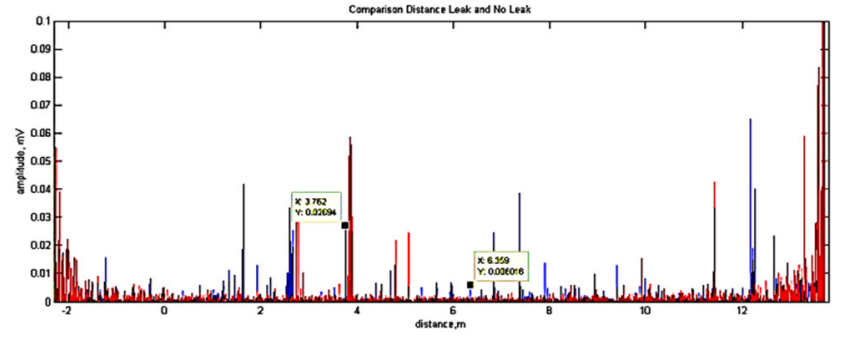

Fig. 9. Pressure signal when opening two leaks

TABLE I. SUMMARY DATA EXPERIMENT BY USING MDPE

\begin{tabular}{|c|c|c|c|c|}
\hline Condition & Features & $\begin{array}{c}\text { Measured } \\
\text { Distance, } \mathbf{m}\end{array}$ & $\begin{array}{c}\text { Analyzed } \\
\text { Distance, } \mathbf{m}\end{array}$ & $\begin{array}{c}\text { Error, } \\
\mathbf{\%}\end{array}$ \\
\hline \multirow{3}{*}{$\begin{array}{c}\text { Open Both } \\
\text { Leak }\end{array}$} & Leak 1 & 1.34 & 1.22 & 8.20 \\
\cline { 2 - 5 } & Leak 2 & 3.99 & 3.79 & 4.94 \\
\cline { 2 - 5 } & Output & 6.15 & 6.05 & 1.67 \\
\hline \multirow{3}{*}{\begin{tabular}{c} 
Open Leak 1 \\
\cline { 2 - 5 }
\end{tabular}} & Leak 1 & 1.34 & 1.46 & 8.25 \\
\cline { 2 - 5 } & Leak 2 & 3.99 & 3.79 & 4.94 \\
\hline \multirow{3}{*}{ Open Leak 2 } & Lutput & 6.15 & 6.33 & 2.88 \\
\cline { 2 - 5 } & Leak 1 & 1.34 & 1.45 & 8.24 \\
\cline { 2 - 5 } & Output & $3 . .99$ & 3.76 & 5.71 \\
\hline
\end{tabular}

\section{CONCLUSION}

The experimental process transient analysis of waves for leakage detection using Wavelet Transform and Cepstrum Analysis is performed. From the result, it shows that the location of the leak can be identified and located from the peak that shows in the graph after being analyzed. The result shows that the accuracy of the leak location is accurate with small error results. To increase the accuracy, there is some recommendation that should be discussed in order to achieve it. From the figures of analyzed result, there are many peaks in the graph. This study only covers the peak that represents the leak part of the pipe. A more experiment should be done in order to differentiate the type of features in the pipe based on the amplitude of the peaks. The second recommendation is that when analyzing the data by using the Wavelet Transform and the Cepstrum Analysis, comparison between filter order should be done and the best filter order should be selected so that the signal that are no needed can be remove and signal from the reflected features is remain in the data.

\section{REFERENCES}

[1] N. F. Adnan, M. F. Ghazali, M. Amin, A. Malik, and A. Ariffin, "Leak detection in MDPE gas pipeline using dual-tree complex wavelet transform," Australian Journal of Basic and Applied Sciences, vol. 8, no. 15 , pp. 356-360, 2014.

[2] N. Adnan, M. Ghazali, M. Amin, and A. Hamat, "Leak detection in gas pipeline by acoustic and signal processing-A review," 2015, vol. 100, no. 1, p. 012013.

[3] T. M. El-Shiekh, "Leak Detection Methods in Transmission Pipelines," Energy Sources, Part A: Recovery, Utilization, and Environmental Effects, vol. 32, no. 8, pp. 715-726, Feb. 2010, doi: $10.1080 / 15567030903058618$.

[4] F. Wang, W. Lin, Z. Liu, S. Wu, and X. Qiu, "Pipeline leak detection by using time-domain statistical features," IEEE Sensors Journal, vol. 17, no. 19, pp. 6431-6442, 2017.

[5] L. Boaz, S. Kaijage, and R. Sinde, "An overview of pipeline leak detection and location systems," 2014, pp. 133-137.

[6] R. Xiao, Q. Hu, and J. Li, "Leak detection of gas pipelines using acoustic signals based on wavelet transform and Support Vector Machine," Measurement, vol. 146, pp. 479-489, 2019.

[7] M. Zadkarami, M. Shahbazian, and K. Salahshoor, "Pipeline leak diagnosis based on wavelet and statistical features using DempsterShafer classifier fusion technique," Process safety and environmental protection, vol. 105, pp. 156-163, 2017.

[8] L. L. Ting, J. Y. Tey, A. C. Tan, Y. J. King, and F. Abd Rahman, "Water leak location based on improved dual-tree complex wavelet transform with soft thresholding de-noising," Applied Acoustics, vol. 174, p. 107751, 2021.

[9] H. M. Yusop, M. Ghazali, M. M. Yusof, and W. W. Hamat, "Improvement of Cepstrum Analysis for the Purpose to Detect Leak, Feature and Its Location in Water Distribution System based on Pressure Transient Analysis/Hanafi. M. Yusop...[et al.]," Journal of Mechanical Engineering (JMechE), no. 4, pp. 103-122, 2019.

[10] N. Motazedi and S. Beck, "Leak detection using cepstrum of crosscorrelation of transient pressure wave signals," Proceedings of the Institution of Mechanical Engineers, Part C: Journal of Mechanical Engineering Science, vol. 232, no. 15, pp. 2723-2735, 2018.

[11] M. F. Lambert, S. T. Nguyen, J. Gong, A. R. Simpson, and A. C. Zecchin, "Leak detection using pseudo random binary sequence excitation and cepstrum analysis," 2017, p. 230.

[12] M. Kothandaraman, Z. Law, M. A. Ezra, and C. H. Pua, "Adaptive Independent Component Analysis-Based Cross-Correlation Techniques along with Empirical Mode Decomposition for Water Pipeline Leakage Localization Utilizing Acousto-Optic Sensors," Journal of Pipeline Systems Engineering and Practice, vol. 11, no. 3, p. $04020027,2020$.

[13] L. Chen, J. Li, Y. Zeng, Y. Chen, and W. Liang, "Magnetic Flux Leakage Image Enhancement using Bidimensional Empirical Mode Decomposition with Wavelet Transform Method in Oil Pipeline Nondestructive Evaluation," Journal of Magnetics, vol. 24, no. 3, pp. 423-428, 2019.

[14] C. Xu, S. Du, P. Gong, Z. Li, G. Chen, and G. Song, “An improved method for pipeline leakage localization with a single sensor based on modal acoustic emission and empirical mode decomposition with Hilbert transform," IEEE Sensors Journal, vol. 20, no. 10, pp. 54805491,2020

[15] M. Shi, H. Zhao, Z. Huang, and Q. Liu, "Signal extraction using complementary ensemble empirical mode in pipeline magnetic flux leakage nondestructive evaluation," Review of Scientific Instruments, vol. 90, no. 7, p. 075101, 2019. 